\title{
O treinamento de resistência variável reduz os níveis de glicemia em idosas
}

\section{octogenárias}

\author{
Variable resistance training reduces blood glucose levels in octogenarian elderly women \\ Entrenamiento de resistencia variable reduce los niveles de glucosa en sangre en mujeres
}

octogenárias

Recebido: 26/09/2021 | Revisado: 03/10/2021 | Aceito: 04/10/2021 | Publicado: 05/10/2021

\author{
Alexandre Medeiros de Quadros Barbosa \\ ORCID: https://orcid.org/0000-0003-4197-252X \\ Faculdade Serra Geral, Brasi \\ E-mail: alexandremedeirosbarbosa@ hotmail.com
}

\begin{abstract}
Resumo
Introdução: O uso de treinamento resistido com resistência variável (TRRV) sobre fatores biodinâmicos tem sido explorado na literatura científica, entretanto informações sobre o uso TRRV em idosos longevos diabéticos ainda são inconclusivas na literatura. Objetivo: Analisar o efeito agudo do treinamento de resistência variável com faixas elásticas sobre os níveis de glicemia de mulheres idosas octogenárias. Método: Sete idosas octogenárias $(81,4 \pm 1,61$ anos de idade) diagnosticadas com diabetes Mellitus tipo dois participaram do estudo. A glicemia de repouso foi aferida 10 minutos antes e 10 minutos após uma sessão de exercícios resistidos com resistência variável utilizando faixas elásticas. Resultados: Após o TRRV os níveis de glicemia (tamanho do efeito: 2,49; p<0,001) reduziram de forma significativa. Conclusão: $\mathrm{O}$ treinamento resistido utilizando resistência variável com faixas elásticas, promoveu redução significante da glicemia de repouso em idosas octogenárias acometidas por Diabetes Mellitus tipo dois.
\end{abstract}

Palavras-chave: Treinamento resistido; Faixas elásticas; Idosas.

\begin{abstract}
Introduction: The use of resistance training with variable resistance (RTVR) on biodynamic factors has been explored in the scientific literature, however information on RTVR use in long-income diabetic elderly is still inconclusive in the literature. Objective: To analyze the acute effect of variable resistance training with elastic bands on blood glucose levels of octogenarian older women. Method: Seven octogenarian older women (81.4 \pm 1.61 years of age) diagnosed with type two diabetes mellitus participated in the study. Resting blood glucose was measured 10 minutes before and 10 minutes after a session of resistance exercises with variable resistance using elastic bands. Results: After the RTVR, blood glucose levels (effect size: 2.49; $\mathrm{p}<0.001$ ) decreased significantly. Conclusion: Resistance training using variable resistance with elastic bands promoted a significant reduction in resting blood glucose in octogenarian women affected by type two Diabetes Mellitus.
\end{abstract}

Keywords: Resistance training; Elastic bands; Elderly.

\section{Resumen}

Introducción: El uso del entrenamiento de resistencia con resistencia variable (ERRV) en factores biodinámicos ha sido explorado en la literatura científica, sin embargo, la información sobre el uso de ERRV en ancianos diabéticos de largos ingresos aún no es concluyente en la literatura. Objetivo: Analizar el efecto agudo del entrenamiento de resistencia variable con bandas elásticas sobre los niveles de glucosa en sangre de mujeres mayores octogenarias. Método: Siete mujeres mayores octogenarias $(81,4 \pm 1,61$ años de edad) diagnosticadas con diabetes mellitus tipo dos participaron en el estudio. La glucemia en reposo se midió 10 minutos antes y 10 minutos después de una sesión de ejercicios de resistencia con resistencia variable utilizando bandas elásticas. Resultados: Después del ERRV, los niveles de glucosa en sangre (tamaño del efecto: 2,49 ; $p<0,001$ ) disminuyeron significativamente. Conclusión: El entrenamiento de resistencia utilizando resistencia variable con bandas elásticas promovió una reducción significativa de la glucosa en sangre en reposo en mujeres octogenarias afectadas por Diabetes Mellitus tipo dos.

Palabras clave: Entrenamiento de resistência; Bandas elásticas; Viejo.

\section{Introdução}

Nas últimas décadas, a expectativa de vida da população aumentou de forma significante, em especial, em países em desenvolvimento como o Brasil (Gomes \& Neto, 2018). Esse fato ocorre devido ao progresso da medicina para o tratamento 
adequado e cura de doenças crônico degenerativas, melhoria nas redes de saneamento básico e melhorias do acesso a informação (Gomes \& Neto, 2018).

Assim, a população idosa tem vivido por um longo período e convivido com doenças crônicas não transmissíveis como a hipertensão arterial e a diabete mellitus por exemplo (Francisco et al., 2018; Almeida et al., 2021). Nesse contexto, a pratica de exercícios físicos tem sido apontada como a principal atividade não medicamentosa para o controle e redução de doenças crônicos degenerativas em especial a diabete mellitus (Boutcher \& Boutcher, 2017; Santiago et al., 2021). Entre as modalidades de exercícios físicos, o aeróbico (de intensidade moderada e o intervalado de alta intensidade) tem sido apontado como uma ferramenta promissora para o controle glicêmico na diabetes mellitus (Da Silva Hortencio, 2018; de Matos et al., 2021).

Nesta perspectiva, anteriormente foi apontado que o treinamento resistido tradicional (i.e., musculação) também promove efeitos benéficos em variáveis hemodinâmicas na população idosa (De Paiva Montenegro, 2015). Entretanto, muitos idosos fragilizados ou dependentes de familiares acabam se privando da prática de exercícios por não poderem se locomover até um ambiente apropriado para a prática, como academias, parques, ginásios e etc (Nathielly \& De Souza, 2020).

Deste modo, anteriormente foi proposto o uso de resistência variável com o auxílio de ligas elásticas para promover adaptações neuromusculares e hemodinâmicas em idosos com idades entre 60 e 70 anos (Jin et al., 2015; De Alencar Silva et al., 2018). Evidenciando, que o método se mostrou promissor e que pode ser uma alternativa para o atendimento domiciliar da população idosa. Entretanto, as informações do uso do método em idosos longevos diabéticos com idade igual ou superior a oitenta anos ainda são inconclusivas na literatura.

Assim, o presente estudo teve por objetivo analisar o efeito agudo do treinamento de resistência variável com faixas elásticas sobre os níveis de glicemia de mulheres idosas octogenárias. A hipótese do estudo é que o treinamento com resistência variável irá promover a redução dos níveis de glicemia em idosas octogenárias.

\section{Metodologia}

\section{Assuntos}

De acordo com Thomas, Nelson e Silverman (2009), o presente estudo é do tipo experimental. A amostra foi recrutada por conveniência composta por sete sujeitos idosos do sexo feminino com média de idade de 81,4 $\pm 1,61$. Para recrutar a amostra foi utilizado de divulgação em redes sociais, assim os familiares que possuíam um parente que se enquadrasse nos critérios de inclusão entraram em contato com o pesquisador responsável por conduzir o experimento. Os critérios de inclusão adotados foram: (1) Ter idade igual ou superior a 80 anos; (2) ser sedentária a no mínimo 6 meses que antecedessem a presente pesquisa; (3) ser diagnosticada clinicamente com Diabetes Mellitus tipo 2 (tendo que apresentar exame médico para comprovação). Como critérios de exclusão adotamos: (1) Possuir lesões musculoesqueléticas ou articulares que impedissem a realização do protocolo de exercícios a ser realizado no presente estudo; (2) Ser usuário de prótese nas articulações do quadril, joelhos ou ombros. (3) Não conseguir concluir o protocolo de exercícios a ser realizado no presente estudo; (4) Não aceitar participar da pesquisa.

\section{Ética}

Todos os voluntários foram informados sobre o estudo e assinaram o Termo de Consentimento Livre e Esclarecido de acordo com a resolução 196/1996 do Conselho Nacional de Saúde, de acordo com os princípios éticos contidos na Declaração de Helsinque, da World Medical Association et al., (2013), e a aprovação ética foi concedida pelo comitê de ética local (Parece: 022093). 


\section{Procedimentos}

\section{Antropometria}

Para fins de caracterização amostral as avaliações antropométrica envolveram medidas de peso, altura e circunferência de quadril, mensuradas três vezes e realizadas de acordo com os protocolos da International Society of the Advancement of Kinanthropometry (Karupaiah, 2018). Assim a média das 3 avaliações foi tomada. A massa corporal foi mensurada em balança digital com precisão de 0,1 kg (FILIZOLA®, São Paulo, Brasil) e a estatura em estadiômetro com precisão de 0,1 cm (SANNY®, São Paulo, Brasil). Para medir a circunferência do quadril, cada participante permaneceu em pé e o avaliador utilizou uma fita métrica (SANNY®, São Paulo, Brasil), a fita foi passada na região média dos glúteos para determinar o menor ponto da circunferência com aproximação de $0,1 \mathrm{~cm}$.

O índice de massa corporal (IMC) foi avaliado de acordo com a seguinte fórmula:

\section{$\operatorname{IMC}_{\left(\mathrm{Kg} / \mathrm{m}^{2}\right)}=$ Peso corporal $_{(\mathrm{Kg})} /\left(\right.$ Estatura $_{(\mathrm{m})} \mathbf{x}$ Estatura $\left._{(\mathrm{m})}\right)$}

IMC: índice de massa corporal. ( $\left.\mathrm{Kg} / \mathrm{m}^{2}\right)$ : Quilogramas por metro quadrado. (Kg): Quilogramas. (m): Metros

\section{Protocolo Experimental}

Inicialmente a amostra foi submetida a uma sessão de familiarização, onde foi realizada uma série de 12 repetições para os seguintes exercícios na respectiva ordem: (1) Agachamento sentando e levantando da cadeira sem uso de carga externa; (2) Abdução de membros inferiores sentado com uso de faixa elástica nos joelhos; (3) Remada baixa sentado na cadeira utilizando faixa elástica; (4) Supino sentado na cadeira utilizando faixa elástica; (5) Flexão plantar em pé; e (6) Flexão de joelhos em pé utilizando faixa elástica. Durante a sessão de familiarização a amostra também foi familiarizada com a escala de percepção subjetiva de esforço CR10- Borg (Williams, 2017). Destaca-se que todos os exercícios foram realizados em uma cadeira de madeira comum com acento acolchoado de 44-cm de altura por 40-cm de largura, com encosto acolchoado de 40$\mathrm{cm}$ de largura por $55-\mathrm{cm}$ de altura (da base do acento ao topo do encosto). Todos os sujeitos foram analisados individualmente em suas residências, nos horários entre 15 e 17h da tarde de acordo com a disponibilidade do participante.

Assim, 48 horas após a sessão de familiarização, antes do início do protocolo de exercícios para fins de caracterização amostral foi realizada a aferição da pressão arterial, frequência cardíaca de repouso, saturação sanguínea. Em seguida, verificamos a glicemia de repouso e posteriormente foi realizada a mesma sequência de exercícios supracitada (com cadência de 2-seg na fase excêntrica e 2-seg na fase concêntrica), os sujeitos realizaram três séries de 12 repetições com intervalos de 30 segundos entre séries (ao todo a sessão durou entre 40 e 60-min). Posteriormente, os níveis de glicemia foram mensurados 10min após o exercício físico. Salientamos, que a Percepção subjetiva de esforço foi perguntada ao final do protocolo experimental.

\section{Resistência Variável}

Para os exercícios utilizamos como resistência variável um kit de faixas elásticas de cor vermelha da marca Theraband® (São Paulo, Brasil). De acordo com Uchida et al., (2016), os elásticos vermelhos da referida marca exercem uma sobrecarga média de 2,61-Kg durante a execução de exercícios. Sendo: 0,65-g a 25\% da sua capacidade elástica; 1,04-kg a $75 \%$ da sua capacidade elástica; $1,60-\mathrm{Kg}$ a $100 \%$ de sua capacidade elástica; $2,08-\mathrm{kg}$ a $175 \%$ de sua capacidade elástica e 2,61 $\mathrm{Kg}$ a $250 \%$ de sua capacidade elástica. Neste sentido, no presente estudo para os exercícios de membros superiores utilizamos a capacidade elásticas das ligas entre $175 \%$ e $250 \%$ e para os membros inferiores entre $75 \%$ e $100 \%$. A capacidade elástica foi calculada com base no comprimento da liga esticada ao máximo sem forçar o elástico (tomado como 100\%), 
posteriormente calculamos o comprimento apropriado para atingir as porcentagens alvos inferiores a $100 \%$ e superiores a $100 \%$.

\section{Análise da Percepção subjetiva de Esforço}

Salientamos que, no intuito de monitorar a intensidade do treinamento, imediatamente após o protocolo de exercícios os avaliados informavam a percepção subjetiva de esforço com base na escala CR10 de Borg (Williams, 2017). A escala consiste em uma reta numérica de zero a dez onde o valor zero corresponde a sem esforço e o valor dez ao esforço máximo (escala CR10-Borg: Sem esforço: 0; Muito, muito leve: 0.5; Muito ligeiramente leve: 1; Pouco leve: 2; Moderado: 3; Um pouco severo: 4; Forte: 5 e 6; Muito severo: 7 e 8; Muito, muito grave quase máximo: 9; Esforço máximo: 10) (Williams, 2017).

\section{Análises Hemodinâmicas}

\section{Frequência Cardíaca e Percentual de Saturação Sanguínea}

Para fins de caracterização amostral a frequência cardíaca (FC) de repouso e o percentual de saturação sanguínea (SpO\%) foram aferidas 10 minutos pré exercícios físicos, por um oxímetro portátil (OLED ${ }^{\circledR}, \mathrm{CMS} 6 \mathrm{D}$, Chicago, USA).

\section{Pressão Arterial}

Também para fins de caracterização amostral a pressão arterial em repouso foi aferida por um único avaliador com treinamento prévio. O sujeito ficou em repouso por 10 minutos, sentado em uma cadeira confortável, com os joelhos posicionados a $90^{\circ}$. A seguir, o avaliador posicionou o manguito do esfigmomanômetro (Premium ®, São Paulo, Brasil) $3 \mathrm{~cm}$ acima da parte anterior da articulação do cotovelo, na sequência a artéria braquial foi identificada. Com o manguito do estetoscópio (3MTM Littmann ®, São Paulo, Brasil) posicionado sobre a artéria, o avaliador insuflou o manguito até a obstrução total da artéria e, a seguir, insuflou mais $25 \mathrm{mmHg}$. Na sequência, foi iniciado o processo de desinsuflação do manguito e pela ausculta da artéria, o avaliador identificou o som do primeiro batimento cardíaco, correspondente à pressão arterial sistólica (PAS) e o som do último batimento cardíaco referindo-se à pressão arterial diastólica (PAD).

\section{Glicemia}

A glicemia foi mensurada 10 minutos antes e 10 minutos após o exercício físico. Utilizamos amostra sanguínea adquirida após uma breve perfuração no dedo indicador direito. A amostra sanguínea foi inserida em um glicosímetro portátil (Accu-Chek Guide®, São Paulo, Brasil) que calculou automaticamente os níveis de glicemia.

\section{Análises Estatísticas}

A normalidade dos dados foi verificada pelos testes de Shapiro-Wilk, assimetria e curtose $(-1,96$ a 1,96$)$ e pelo coeficiente de variação (CV\%) (normalidade aceita quando CV\% <30\%). O pressuposto de normalidade não foi negado por nenhum dos testes utilizados. Deste modo, os dados foram expostos de forma descritiva por meio da média e desvio padrão. As comparações entre os níveis de glicemia [pré e pós exercícios] foram realizadas pelo teste "T dependente de Student". O tamanho do efeito e seus respectivos intervalos de confiança de 95\% (IC95\%) foram calculados pelo d-Cohen. Para interpretar o d-Cohen, adotamos a magnitude: Pequeno: 0,20 a 0,49; Médio: 0,50 a 0,79; Largo: 0,80 a 1,19; Muito Largo: >1,20 (Cohen, 2013). Todas as análises foram feitas no software gratuito JASP® (Versão 0,14,1/18/ 2020, Tóquio, Japão), considerando a significância de $\mathrm{p}<0.05$. 


\section{Resultados}

A Tabela 1 expõe as características da amostra estudada, as idosas octogenárias estavam com IMC na classificação sobrepeso (de 25.0 a 29.9) e pressão arterial em níveis normais. Além disso, todos os sujeitos da amostra relataram ter grau de escolaridade com nível superior completo e possuíam renda mensal >3 salários mínimos brasileiros. Para o controle da diabetes mellitus todas as idosas usavam o medicamento Metformina (500mg 2 x dia) e faziam acompanhamento nutricional com um profissional de nutrição. Destacamos, que em relação a percepção subjetiva de esforço (PSE) aferida após o exercício físico, a amostra apontou valores entre a PSE 2 e 4, indicando que a intensidade percebida dos exercícios foi entre pouco leve (PSE 2) e pouco severo (PSE 4).

Tabela 1. Caracterização amostral.

\begin{tabular}{|c|c|}
\hline Variáveis & Média \pm Desvio Padrão \\
\hline Idade $_{\text {(Anos) }}$ & $81,4 \pm 1,61$ \\
\hline $\operatorname{Peso}_{(\mathrm{Kg})}$ & $70,8 \pm 3,84$ \\
\hline Estatura $(\mathrm{m})$ & $1,66 \pm 0,05$ \\
\hline Quadril $(\mathrm{cm})$ & $101,5 \pm 5,41$ \\
\hline $\mathrm{IMC}_{\left(\mathrm{Kg} / \mathrm{m}^{2}\right)}$ & $25,5 \pm 1,82$ \\
\hline Pressão Arterial Sistólica $(\mathrm{mmHg})$ & $127,1 \pm 13,8$ \\
\hline Pressão Arterial Diastólica (mmHg) & $77,4 \pm 5,85$ \\
\hline Frequência Cardíaca de repouso (Bpm) & $102,7 \pm 9,82$ \\
\hline Saturação Sanguínea (\%) & $96,0 \pm 1,00$ \\
\hline PSE (CR10-Borg) & $2,85 \pm 0,69$ \\
\hline
\end{tabular}

IMC: Índice de Massa Corporal. IAC: Índice de Adiposidade Corporal. Kg: Quilogramas. (m): Metros. (cm): centímetros. $\left(\mathrm{Kg} / \mathrm{m}^{2}\right)$ : Quilogramas por metro quadrado. (\%): Percentual. (mmHg): Milímetros de mercúrio. (Bpm): Batimentos por minuto. PSE: Percepção subjetiva de esforço. (CR10-Borg): Escala de percepção subjetiva de esforço de 10 pontos proposta por Borg. Fonte: Autores.

A Figura 1, mostra os resultados dos níveis de glicemia nas condições pré e após exercícios. Evidenciando que os níveis de glicemia reduziram de forma significante (Tamanho do efeito: 2,49; IC95\%-Tamanho do efeito: [0,90; 4,00]).

Figura 1. Comparações dos níveis de glicemia nos momentos pré e pós exercícios.

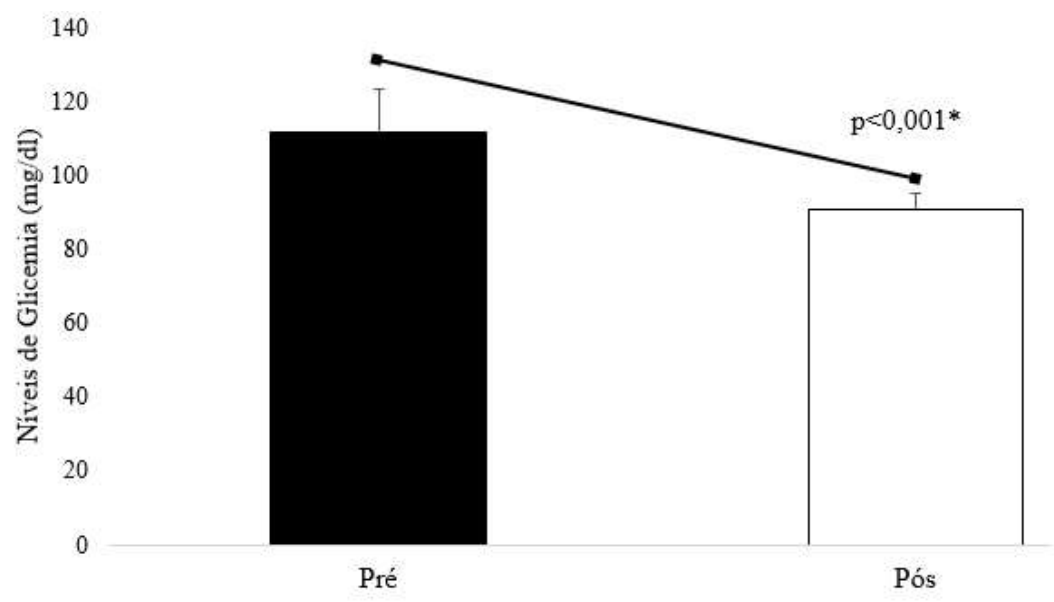

(mg/dl): Miligramas por decilitro. Fonte: Autores. 


\section{Discussão}

O presente estudo teve por objetivo analisar o efeito agudo do treinamento de resistência variável com faixas elásticas sobre os níveis de glicemia de mulheres idosas octogenárias. Desta forma, o principal achado apontou que em um grupo composto por idosas octogenárias, o treinamento com resistência variável em intensidade moderada (PSE >2 e <4) utilizando faixas elásticas promoveu efeito agudo em relação a homeostase dos níveis de glicemia sanguínea. Sabe-se que o exercício físico em intensidades baixa e moderada é capaz de promover a manutenção da saúde cardiovascular, e de promover alterações benéficas na pressão arterial e frequência cardíaca de pessoas idosas (Souza et al., 2019; Bellafiore et al., 2019; Hughes, Ueda \& Casey, 2016; Altavilla \& D'ella, Raiola, 2018; Moreira et al., 2016).

Deste modo, observou-se no presente estudo que para os níveis de glicemia os resultados também foram benéficos, assim, o presente estudo corroborou com os achados de Jin et al., (2015), os autores apontaram que o treinamento com faixas elásticas realizado duas vezes por semana por um período longitudinal de 12 semanas, foi capaz de reduzir os níveis de glicemia sanguínea em mulheres idosas acometidas por hiperglicemia. Em convergência com os dados da presente pesquisa, De Alencar Silva et al., (2018) identificaram que de forma crônica (intervenção de 12 semanas) o treinamento com resistência variada utilizando faixas elásticas, foi capaz de promover adaptações neuromusculares que ampliaram a força muscular, redução dos níveis de glicemia em repouso, redução da resistência à insulina e melhora da funcionalidade motora em um grupo comporto por sete idosos acometidos por sarcopênia (70,7 $\pm 8,0$ anos de idade, três homens e quatro mulheres).

Os efeitos do exercício físico sobre a glicemia podem ser justificados porque durante a pratica de exercícios o tecido musculoesquelético irá precisa de energia para se mover, e essa energia será adquirida através das reservas de glicose tanto em vias oxidativas como em vias glicolíticas (Gomes \& Neto, 2018). Em adição, para evitar um quadro hiperglicêmico, que ocorrem em alguns sujeitos devido ao fato do organismo não está habituado a usar altas taxas glicose como energia durante o exercício é recomendado utilizar intensidades moderadas (De Paiva Montenegro, 2015). Neste sentido é recomendado monitorar a percepção subjetivas de esforço dos sujeitos durante o exercício físico (Williams, 2017).

Diante da perspectiva, o presente estudo traz como ponto forte, o fato dos achados demonstra que uma única sessão de treinamento resistido com faixas elásticas foi capaz de promover adopções agudas benéficas para os níveis de glicemia em idosas octogenárias. Deste modo, os achados do presente estudo sugerem que a aplicação do treinamento resistido com faixas elástica pode ser vantajoso para aplicação clínica em idosos frágeis, em programas de reabilitação (i.e., pacientes acamados em hospitais) ou para o atendimento a domicílio de idosos saudáveis que optam por se exercitarem em casa. Destacamos que as faixas elásticas são portáteis e exigem o uso de pouco espaço e possuem um menor custo financeiro em relação a outros equipamentos utilizados para treinamento resistido (i.e., halteres, anilhas, etc).

Contudo, apesar da relevância dos resultados o presente estudo possui como principal limitação o fato de ter utilizado uma pequena amostra de idosas octogenárias, logo, em grupos com outras faixas de idade e ou sexo os resultados podem divergir. Deste modo, sugerimos que estudos posteriores repitam o experimento em grupos com características distintas as da amostra do presente estudo.

\section{Conclusão}

Com base nos resultados do presente estudo, conclui-se que um protocolo agudo de treinamento resistido utilizando resistência variável com faixas elásticas, promoveu redução significante nos níveis de glicemia em idosas octogenárias. Neste sentido, sugerimos que futuros estudos investiguem de forma aguda ou crônica o efeito do treinamento resistido sobre outras variáveis hemodinâmicas como a pressão arterial de repouso, frequência cardíaca de repouso e a variabilidade da frequência cardíaca em idosos de ambos os sexos. 


\section{Referências}

Almeida, G. D. O., Aidar, F. J., Matos, D. G. D., Almeida-Neto, P. F. D., Melo, E. V. D., Barreto Filho, J. A. S., \& Sobral Sousa, A. C. (2021). Non-Targeted Self-Measurement of Blood Pressure: Association with Self-Medication, Unscheduled Emergency Visits and Anxiety. Medicina, 57(1), 75.

Altavilla, G., D'Elia, F., \& Raiola, G. (2018). A brief review of the effects of physical activity in subjects with cardiovascular disease: An interpretative key. Sport Mont, 16(3), 103-106.

Bellafiore, M., Battaglia, G., Bianco, A., \& Palma, A. (2019). Expression pattern of angiogenic factors in healthy heart in response to physical exercise intensity. Frontiers in physiology, 10, 238.

Boutcher, Y. N., \& Boutcher, S. H. (2017). Exercise intensity and hypertension: what's new? Journal of human hypertension, 31(3), 157-164.

Cohen, J. (2013). Statistical power analysis for the behavioral sciences. Academic press.

Da Silva Hortencio, M. N., da Silva, J. K. S., Zonta, M. A., de Melo, C. P. A., \& França, C. N. (2018). Efeitos de exercícios físicos sobre fatores de risco cardiovascular em idosos hipertensos. Revista Brasileira em Promoção da Saúde, 31(2).

De Alencar Silva, B. S., Lira, F. S., Rossi, F. E., De Freitas, M. C., Freire, A. P. C. F., Dos Santos, V. R., \& Gobbo, L. A. (2018). Elastic resistance training improved glycemic homeostasis, strength, and functionality in sarcopenic older adults: a pilot study. Journal of exercise rehabilitation, $14(6)$, 1085.

de Matos, D. G., de Almeida-Neto, P. F., Moreira, O. C., de Souza, R. F., Tinoco Cabral, B. G. D. A., Chilibeck, P., \& Aidar, F. J. (2021). Two Weekly Sessions of High-Intensity Interval Training Improve Metabolic Syndrome and Hypertriglyceridemic Waist Phenotype in Older Adults: A Randomized Controlled Trial. Metabolic Syndrome and Related Disorders.

De Paiva Montenegro, L. (2015). Musculação para a qualidade de vida relacionada à saúde de hipertensos e diabéticos tipo 2 . Revista Brasileira de Prescrição e Fisiologia do Exercício (RBPFEX), 9(51), 105-109.

Francisco, P. M. S. B., Segri, N. J., Borim, F. S. A., \& Malta, D. C. (2018). Prevalência simultânea de hipertensão e diabetes em idosos brasileiros: desigualdades individuais e contextuais. Ciência \& Saúde Coletiva, 23, 3829-3840.

Gomes, I. C.; Neto, L. D. O. (2018). Saúde do idoso: um olhar de profissionais de educação física. Lura Editorial.

Hughes, W. E., Ueda, K., \& Casey, D. P. (2016). Chronic endurance exercise training offsets the age-related attenuation in contraction-induced rapid vasodilation. Journal of applied physiology, 120(11), 1335-1342.

Jin, E. H., Park, S., \& So, J. M. (2015). The effect of muscle power training with elastic band on blood glucose, cytokine, and physical function in elderly women with hyperglycemia. Journal of exercise nutrition \& biochemistry, 19(1), 19.

Karupaiah, T. (2018). Limited (ISAK) profiling the International Society for the Advancement of Kinanthropometry (ISAK). Journal of Renal Nutrition and Metabolism, 3(1), 11-11.

Moreira, S. R., Cucato, G. G., Terra, D. F., \& Ritti-Dias, R. M. (2016). Acute blood pressure changes are related to chronic effects of resistance exercise in medicated hypertensives elderly women. Clinical physiology and functional imaging, 36(3), 242-248.

Nathielly, M., \& de Souza, S. D. (2020). Exercícios Terapêuticos domiciliares em idosos fragilizados Revisão de Literatura. Anais do EVINCI-UniBrasil, 6(1), $157-157$.

Santiago, M. S., Aidar, F. J., dos Santos Moraes, T. L., de Farias Neto, J. P., Vieira Filho, M. C., de Vasconcelos, D. P., \& da Silva Júnior, W. M. (2021). The Management between Comorbidities and Pain Level with Physical Activity in Individuals with Hip Osteoarthritis with Surgical Indication: A Cross-Sectional Study. Medicina, 57(9), 890.

Souza, D., Barbalho, M., Vieira, C. A., Martins, W. R., Cadore, E. L., \& Gentil, P. (2019). Minimal dose resistance training with elastic tubes promotes functional and cardiovascular benefits to older women. Experimental gerontology, 115, 132-138.

Thomas, J. R., Nelson, J. K., \& Silverman, S. J. (2009). Métodos de pesquisa em atividade física. Artmed Editora.

Uchida, M. C., Nishida, M. M., Sampaio, R. A. C., Moritani, T., \& Arai, H. (2016). Thera-band® elastic band tension: reference values for physical activity. Journal of Physical Therapy Science, 28(4), 1266-1271.

Williams, N. (2017). The Borg rating of perceived exertion (RPE) scale. Occupational Medicine, 67(5), 404-405.

World Medical Association. (2013). World Medical Association Declaration of Helsinki: ethical principles for medical research involving human subjects. Jama, 310(20), 2191-2194. 